\title{
Multimedia augmented m-learning: Issues, Trends and Open Challenges
}

\author{
Abdullah Yousafzai ${ }^{1,2, *}$, Victor Chang ${ }^{3}$, Abdullah Gani $^{1,2,{ }^{* *},}$, Rafidah Md Noor ${ }^{1}$ \\ ${ }^{1}$ Department of Computer System and Technology, University of Malaya (UM), 50603 Kuala Lumpur, Malaysia \\ ${ }^{2}$ Center for Mobile Cloud Computing Research, University of Malaya (UM), 50603 Kuala Lumpur, Malaysia \\ ${ }^{3}$ Xi'an Jiaotong Liverpool University, Suzhou, China \\ *Correspondence Email: abdullahyousafzai@ siswa.um.edu.my, Tel: +6073967786 \\ ${ }^{* * *}$ Correspondence Email: abdullah@um.edu.my, Tel: +60129156105
}

\begin{abstract}
The advancement in mobile technology and the introduction of cloud computing systems enable the use of educational materials on mobile devices for a location- and time-agnostic learning process. These educational materials are delivered in the form of data- and compute-intensive multimedia-enabled learning objects. Given these constraints, the desired objective of mobile learning (m-learning) may not be achieved. Accordingly, a number of m-learning systems are being developed by the industry and academia to transform society into a pervasive educational institute. However, no guideline on the technical issues concerning the m-learning environment is available. In this study, we present a taxonomy of such technical issues that may impede the life cycle of multimedia-enabled m-learning applications. The taxonomy characterizes the constraint regarding mobile device heterogeneity issues, network performance issues, content heterogeneity issues, content delivery issues, and user expectation. These issues are described, along with their causes and measures, to achieve solutions. Furthermore, we have identified five trending areas through which the adaptability and acceptability of multimedia-enabled m-learning platforms can be increased. Finally, we conclude the article by briefly discussing five open challenges, namely, low complexity encoding, data dependency, measurement and modeling, interoperability, and security.
\end{abstract}

\section{Keywords: Mobile Learning; Cloud Learning; Multimedia-enabled Learning; Personalized Learning}

\section{INTRODUCTION}

The enhancement of new technologies in wireless communication systems as well as smartphone hardware and software (operating system) enabled the deployment of sophisticated communication and computation infrastructures to deliver various multimedia-oriented mobile services. In these multimedia services, their generated multimedia contents/data have common continuous-, data-, and compute-intensive qualities. In general, multimedia contents are bounded by strict timing relationships. These timing relationships must be obeyed when processing and rendering such data (e.g., transmitted video, displayed video, rendered 3D graphics, or played out audio).

The intensive penetration of smart mobile devices and mobile applications has fueled a new wave of demand for mobile services, such as mobile learning (m-learning), which introduces the wireless and ubiquitous system of learning. With the assistance of such systems, smartphone users can use the educational material on their device to learn. Such educational materials can be in the form of multimedia content or learning objects, which are more interactive, highly visual, engaging, and effective for learner quality of learning (QoL). Indeed, these learning objects can be easily used by the students whenever necessary and wherever they are by maximizing this "always there, always on" technology (Ally, 2009). Consequently, the subscribers to the learning system not only expect ubiquitous network connections but further demand to seek a rich media experience wherever they go and whenever necessary. In fact, this overwhelming thriving of mobile media gives rise to multifold technical challenges. These problems include (i) the difficulty in sustaining the satisfactory quality of experience (QoE)/QoL over inherently unreliable wireless links and (ii) the tendency of large simultaneous delivery of media contents tends to swamp existing mobile network infrastructure. Moreover, a fundamental gap exists between the resource demands of computationally expensive 
processing of multimedia contents and the capabilities of battery-constrained mobile devices. In this context, the current development in mobile cloud computing (MCC) seems to bridge this gap in terms of providing mobile applications the capabilities of cloud servers and storage together with connectivity.

Cloud computing offers on-demand and elastic delivery of software and hardware infrastructures via the Internet using a service-oriented model (AFox et al., 2010). Cloud computing emerges because of advantages, such as reduction of the capital cost, decoupling technological fabric from service delivery, and providing mobility and flexibility of stored data. Mobile cloud computing is actually the amalgamation of the mobile environment with the cloud computing systems. Mobile cloud computing is conceived to mitigate the issues related to mobile device performance (battery life, processing time, and storage), mobile environment (heterogeneity, availability, and scalability), and security (privacy and reliability). By resorting to the cloud as a reservoir for additional computation and storage, mobile media services can scale better with user demand because of their flexibility and dynamism at relatively low costs.

Furthermore, mobile cloud computing can provide an infrastructure for scalable delivery of various mobile multimedia applications, such as m-learning, mobile gaming, and interactive video streaming (Victor Chang, Walters, \& Wills, 2015). For example, mobile cloud computing concepts and technologies can enhance the desktop-based webfacilitated or traditional classroom-based educational experiences by offloading the personalized translating and transformation of the learning material to the cloud from the subscribers' devices. Furthermore, in the large-scale location and time-agnostic m-learning environment, a distributed cloud environment in conjunction with the mobile cloud can provide various multimedia services to the subscriber of the m-learning environment. These services may include pre-composed multimedia lectures, videos, animations, and images. However, the retrieval and communication of pre-orchestrated and real-time multimedia data impose challenges of heterogeneous networks, quality of service (QoS) requirements, and heterogeneous multimedia data on the design of the MCC architecture (Felemban, Basalamah, \& Ghafoor, 2013).

In recent times, the smartphones capabilities have significantly improved; however, when compared with the tradition desktop computers they are still far behind. Furthermore, the introduction of cloud-based mobile media has further posed various limitations due to heterogeneious mobile devices and dynamics of other technologies on the applications in which they are being utilized. Consequently, providing a generic solution for these limitations for all types of mobile environments; therefore, special attention is required when developing such mobile multimedia learning applications. To mitigate the heterogeneity problem for a wide range of mobile devices, the multimedia content objects should be agnostic of mobile device software and hardware (Ahmad \& Gabbouj, 2008). Furthermore, recent years witnessed a significant growth in the total of multimedia coding standards and the number of delivery protocols for multimedia contents (Deursen et al., 2009). In fact, the diverse mobile phone architectures and characteristics, multimedia coding standards, delivery protocols, intrinsic characteristics of the wireless networks and cloud-based multimedia service delivery create several issues. These concerns may include cloud resource provisioning and scheduling, media content management and delivery, QoS, user mobility, and security. Furthermore, the service providers may pool the statistics of mobile devices in the same region and leverage such knowledge to improve their media transport and caching strategies. Apart from this attempt, mobile applications may offload computationally expensive tasks to the cloud.

This paper aims to outline the mobile multimedia cloud computing issues in the m-learning environment and provide insights on these issues, which will be useful when designing the m-learning system. The remainder of the article is organized into the following five sections: Section II presents the background on m-learning system and education as a service (EaaS) with its characteristics and advantages. Section III presents the taxonomy of issues related to mlearning environments. Section IV presents several trending areas, which can increase the adaptability and acceptability of multimedia-enabled m-learning systems. Section V discusses several open research opportunities, which should be addressed to stabilize and improve the m-learning environments. Finally, Section VI concludes the article. 


\section{BACKGROUND}

In this section, we describe the m-learning environments by exploring how the cloud can benefit m-learning using EaaS.

\section{a) Mobile Learning}

The ever-increasing functional capabilities transformed the smart mobile devices into ubiquitous computing devices. This development probably led to the incubation of new m-services into the spectrum of the mobile environment, services offers, provisioning and consumption of audiovisual formats, context, and localization-based information dissemination, and user preference-based personalized assistance (Victor Chang, 2016; Herrington, Herrington, \& Mantei, 2009; Sharples \& Roschelle, 2010). These type services consumed by end user mobile devices can be utilized for learning purposes to improve desktop/web-facilitated or conventional classroom based educational process. This possibility has led to research on educational initiatives to investigate the potential and effect of the learning contents delivered via ubiquitous mobile devices. Generally, "m-learning" is the process of development, delivery, and consumption of learning material via a learning system subscriber using mobile devices. Figure 1 shows the schematics of m-learning in conjunction with distance and e-learning.

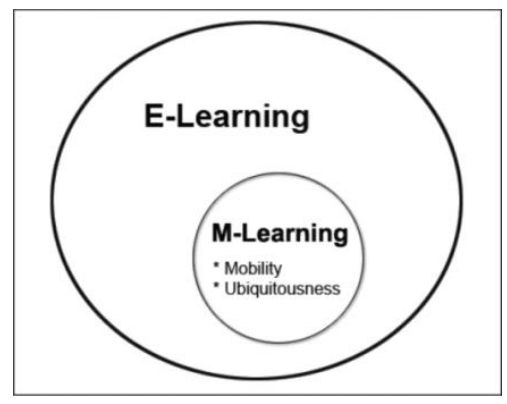

Figure 1 Schematic of Mobile Learning

In fact, m-learning is a form of distance education system delivered on a mobile device. However, m-learning is distinctive in its application through its emphasis on the learning across contexts (location, time and other environmental factors), differentiated through mobile devices. One can simply define m-learning as a learning process in which the learner does not remains at a fix location and benefits the learning prospects accessible via smart mobile devices (Alabbadi, 2011; Chen, Liu, Han, \& Xu, 2010; Gao \& Zhai, 2010; Kitanov \& Davcev, 2012; Masud \& Huang, 2013). Consequently, m-learning reduces the constraints of location bounded learning, given the flexibility and mobility of smart mobile devices. A generic m-learning environment is presented in Figure 2.

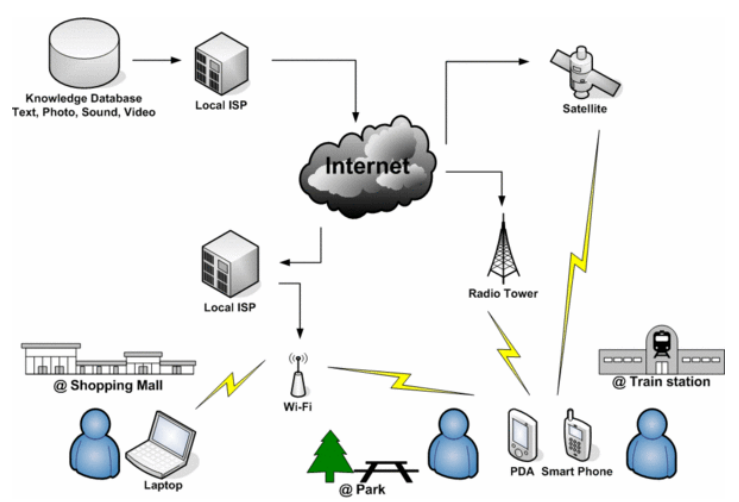

Figure 2 An m-Learning Environment ${ }^{1}$

\footnotetext{
${ }^{1}$ http://www.lshtm.ac.uk/edu/elearning/technologies/mobile/
} 
The suitability of m-learning lies in its nearly complete accessibility from practically anywhere in the globe, provided the presence of network connectivity. M-learning (e.g., e-learning) is also collaborative, resulting in the availability of immediate feedback and instructions from the instructor and peers. Furthermore, m-learning is portable in that it replaces paper-based learning materials with personalized digital learning contents. Furthermore, utilizing the mlearning ideology is simple for a more entertaining and effective learning experience. With smart mobile devices, learners have easy access to educational contents. They employ their devices as an augmentation tool for their educational needs and access the different educational materials, such as essays, articles, diagrams, and other academic materials that can enhance their performance in or out of the classroom.

In a traditional classroom-based learning environment, the student probably becomes nervous when an instructor calls upon him or her, imagining that he or she could be in trouble. With mobile-based learning, the interaction between student and teacher is free from worry or anxiety. Using m-learning, even shy students interact more openly with his teacher. An instructor may also employ mobile devices to communicate with students requiring special attention or learning method. Several educationalists (Ally \& Tsinakos, 2014; Ghazizadeh, 2012; Godwin-jones, 2011) believe that every learner requires a different pedagogy or strategy to absorb the learning material. Through m-learning, students can learn in their own personalized methods and overcome the distance and expense issues. Finally, mlearning assists students with learning disabilities, considering the numerous m-learning applications targeting the students with physical impairments and learning disabilities; m-learning can be said to create opportunities for students with special needs to have an equal platform compared with those regular students.

Now, considering all the mentioned applications and advantages of m-learning methods, the main learning material in the current spectrum of modern mobile devices is via audiovisual learning elements. These media-enabled learning elements are data- and compute-intensive. Conversely, the capabilities of smart mobile devices are still restricted by their battery life. The smart mobile devices generally drain battery quickly when performing operations on multimedia contents for a longer time span (which maps to the time span of a lecture). In such a scenario, mobile cloud computing can mitigate this issue by offloading the processing to the cloud and presentation on the learner device to extend battery life. Another approach would be to compose special services, which can be readily utilized by the m-learning environment to ensure a seamless m-learning experience. Such a service regarded as EaaS is described in the subsequent subsection.

\section{b) Education as a Service}

Cloud computing is a business model introducing a XaaS service delivery model while simultaneously charged using an on-demand pay-as-you-go method and deployed across different deployment models (Mell \& Grance, 2011). Cloud computing systems revolutionize the business life cycle by reducing the capital investment in infrastructure while maintaining additional focus on business services and strategies. Given these eminent features, the Joint Information Systems Committee (JISC) has recommended cloud services for research and educational activities ${ }^{2}$. Furthermore, to provision EaaS, cloud building block services are orchestrated or a dedicated education services are developed and delivered using a software/platform as a service model (He, Cernusca, \& Abdous, 2011). This educational service is regarded as EaaS, which is a combination of computational resources, applications, protocols, and delivery models, along with pedagogical/learning resources (Wheeler \& Waggener, 2009). Noticeably, m-learning is essentially elearning as illustrated in the schematic of m-learning presented in Figure 1. Basically, m-learning can further maximize advantage of the EaaS over the cloud, along with the concepts of mobile cloud and mobile edge computing. The effect of cloud computing on the learning process delivered via EaaS is further validated using a supply chain business model

\footnotetext{
2 ("Cloud computing increasingly attractive to universities" ) https://www.jisc.ac.uk/news/cloud-computing-increasingly-attractive-to-universities-says-jisc-16-may-2011
} 
(Victor Chang \& Wills, 2013). The authors further introduce the organizational sustainability model (Victor Chang, Walters, et al., 2015) to measure the QoL for evaluating and reviewing the EaaS projects and services.

Orchestration and development of EaaS for the m-learning environment have its own special needs because of the limitation of the mobile device and diversity of the learning contents (Juan \& Jun, 2013; Liu, 2013; Masud \& Huang, 2012, 2013). Addressing these special needs when orchestrating an EaaS service can improve the overall learning process of the subscribers. These special technical needs are captured and described using taxonomy in the subsequent section.

\section{TAXoNomy OF ISSUES IN MobILE MULTIMEdiA-ENABLED MobILE LEARNING}

Multimedia applications, such as m-learning applications, tackle various issues because of the intensive nature of data. In Figure 3, we presented these technical issues using an issue-based taxonomy and provide a brief description pertaining to the issues and their underlying causes.

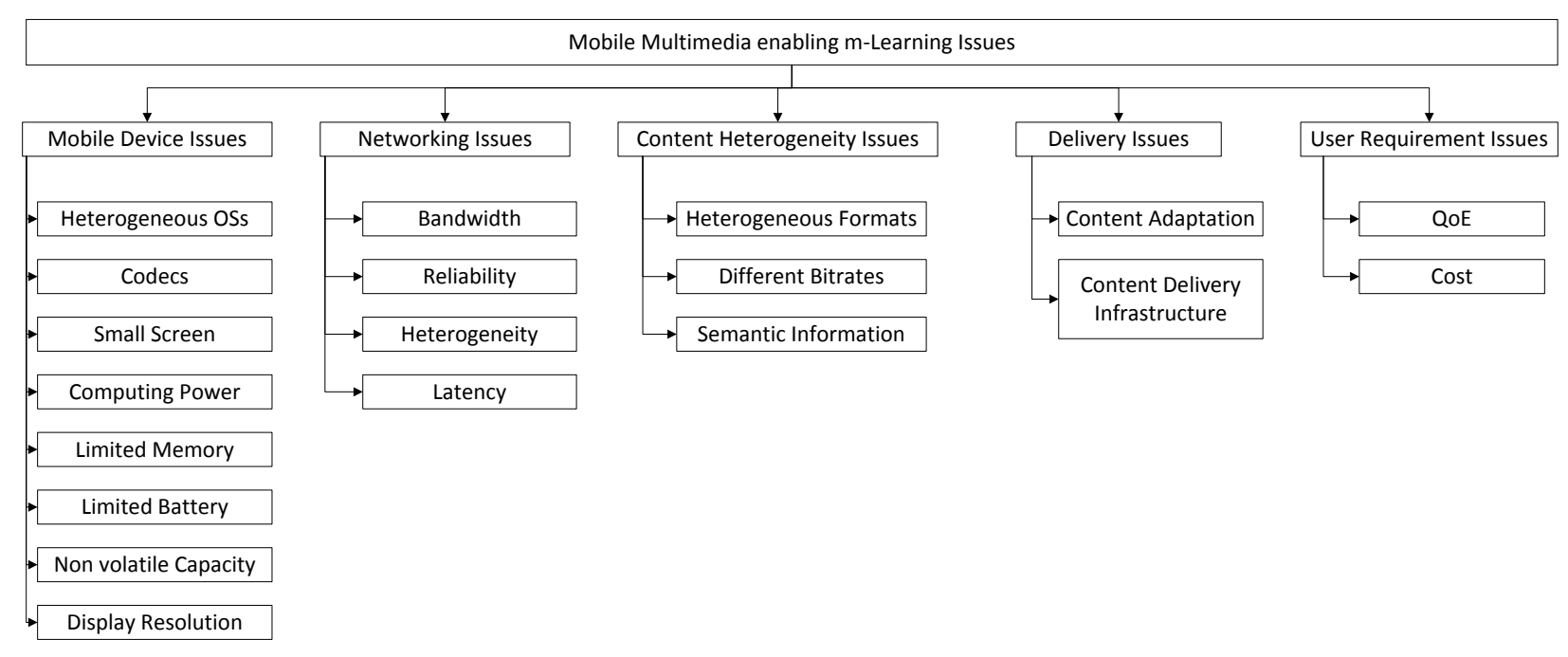

Figure 3 Taxonomy of Issues in Mobile Multimedia Cloud Computing

\section{Mobile Device Issues}

The components of mobile device constraints, as depicted in Figure 3, are due to the small form factor of mobile devices; the number and the potential of mobile device resources are limited, such as low battery life, storage, input methods, and screen sizes. The existing battery technology is not developing quickly (or accordingly) compared with the rapid evolution of the memory and CPU power. The transmission and processing of multimedia contents deplete battery quickly. Consequently, the limited battery necessitates the design of energy-efficient protocols and algorithms for multimedia transmission and processing in the mobile environment. Another issue is device heterogeneity due to different software (operating systems) and hardware architectures. Furthermore, mobile devices strongly vary in screen resolutions, screen sizes, and encoding and decoding mechanisms. Thus, the same multimedia content should be processed/tailored transparently and optimally for the users according to the functional and systematic characteristics of mobile devices (Luo \& Shyu, 2011).

\section{Networking Issues}

The dynamics of the wireless networks shown in Figure 3, which affect the behavior of a multimedia-enabled mlearning application, the QoL, and the QoE, are depicted; below is a brief discussion on these issues: 
a) Low Bandwidth: Low bandwidth is one of the challenging problems, which may deteriorate the QoS of any mobile multimedia application, given that the bandwidth in wireless radio networks is significantly scarcer than in wired networks. The fluctuation in the bandwidth due to weather and other conditions further increases the complexity of the issue. Hopefully, this limitation will be mitigated with the imminent deployment of modern wireless networks but not completely eliminated as the video bit rate also increases monotonically with time.

Table 1 shows the bandwidth requirement of a typical H.264 (Wiegand, Sullivan, Bjontegaard, \& Luthra, 2003) encoded video stream with different settings.

Table 1 Video Resolution Bandwidth ${ }^{3}$

\begin{tabular}{|llll|}
\hline Video codec & Resolution & $\begin{array}{l}\text { Maximum video payload } \\
\text { bitrate }(\mathbf{K b p s})\end{array}$ & $\begin{array}{l}\text { Minimum video payload } \\
\text { bitrate (Kbps) }\end{array}$ \\
\hline H.264 & $320 \times 180$ & 250 & 15 \\
\hline H.264/RTVideo & $424 \times 240$ & 350 & 100 \\
\hline H.264 & $480 \times 270$ & 450 & 200 \\
\hline H.264/RTVideo & $640 \times 360$ & 800 & 300 \\
\hline H.264 & $848 \times 480$ & 1500 & 400 \\
\hline H.264 & $960 \times 540$ & 2000 & 500 \\
\hline H.264/RTVideo & $1280 \times 720$ & 2500 & 700 \\
\hline H.264 & $1920 \times 1080$ & 4000 & 1500 \\
\hline H.264/RTVideo & $960 \times 144$ & 500 & 15 \\
\hline H.264 & $1280 \times 192(20: 3)$ & 1000 & 250 \\
\hline H.264 & $1920 \times 288(20: 3)$ & 2000 & 500 \\
\hline
\end{tabular}

b) Network Reliability: Reliability of the wireless network is an important issue given that the QoE of multimediaenabled m-learning applications strongly depends on the long-lasting network connectivity with an acceptable bandwidth, packet loss, jitter, and delay. A mobile learner depends on the access network and may possibly be prevented from connecting to the learning system provider because of flow congestion and failures, which are habitual with wireless radio networks. This disconnection is recurrent in places, such as subways and tunnels, and providing a reliable wireless connectivity that is scalable and cost efficient while the learner is commuting is a challenge.

c) Heterogeneity: The users behind the smart handheld devices can access the multimedia-enabled learning platform via a range of radio interfaces available on the device, such as GPRS, Wi-Fi, WiMax, and LTE. In such heterogeneous network availability, deploying an efficient connectivity algorithm on the m-learning client and server platform becomes extremely important, which allows the users to permanently stay connected as well as seamlessly and transparently switch established network connections from one interface to another while staying connected to the server-side learning environment.

d) High Access Latency: Longer access latencies in wireless radio networks are a major barrier faced by mobile multimedia-enabled m-learning applications. Longer delays and connection interruption significantly deteriorate the QoL and QoE of the mobile learner. Humans are sensitive to this quality deterioration caused by jitter and longer delays that are extremely difficult to regulate in the wireless and WAN networks. Conversely, bandwidth is significantly enhanced in modern access networks; latency is unlikely to improve (Bourguiba, Agha, \& Haddadou, 2012).

\section{Contents Heterogeneity}

The heterogeneity of the multimedia contents is due to its spatiotemporal characteristics. The higher the spatial resolution, the finer the available details; in the case of video, the higher the temporal resolution (frames per second), the smoother the video (Knoche, McCarthy, \& Sasse, 2005). These resolutions place a significant effect on the size and bit rate of the multimedia contents. The potential effects are higher storage, networking, and processing costs.

\footnotetext{
${ }_{3}^{3}$ ("Network Bandwidth Requirements for Media Traffic") http://technet.microsoft.com/en-us/library/jj688118.aspx
} 
Nevertheless, multimedia-enabled learning contents are compressed for efficient storage and faster transmission. Alternatively, different smart mobile device platforms are supporting varying multimedia storage formats and encoding/decoding standards with a significant effect on the content itself and its delivery procedure to no compliant device. For example, considering video coding standards, Android-based smart devices support MPEG-4 SP, AVC, H.264, H.263, and VP8, whereas iOS supports H.264 and MPEG-4. Evidently, transcoding techniques are obligatory for seamless and transparent adaptation and delivery of the learning material to enable interoperability and support a large number of mobile devices (Kovachev, Cao, \& Klamma, 2011). Moreover, another challenge is the processing of semantically annotated multimedia contents. Table 2 clearly depicts the heterogeneity in terms of the resolution standards, compression formats, bit rate, size, CPU usage, and quality.

Table 2 Heterogeneity of Multimedia Contents ${ }^{4}$

\begin{tabular}{|lll|llllll|} 
Resolution & $\mathbf{3 5 2 x 2 4 0}$ & $\mathbf{4 8 0 x 4 8 0}$ & $\mathbf{7 2 0 x 4 8 0}$ & $\mathbf{1 9 2 0 x 1 0 8 0}$ & $\mathbf{6 4 0 x 4 8 0}$ & $\mathbf{6 4 0 x 4 8 0}$ & $\mathbf{3 2 0 x 2 4 0}$ & $\mathbf{7 2 0 x 4 8 0}$ \\
\hline $\begin{array}{l}\text { Compression } \\
\text { Format }\end{array}$ & MPEG1 & MPEG2 & $\begin{array}{l}\text { MPEG2, } \\
\text { MPEG1 }\end{array}$ & $\begin{array}{l}\text { MPEG2 } \\
\text { (WMV- } \\
\text { MPEG4) }\end{array}$ & MPEG4 & $\begin{array}{l}\text { Sorenson, } \\
\text { Cinepak, } \\
\text { MPEG4 }\end{array}$ & RM & DV \\
\hline Video bitrate & $1150 \mathrm{kbps}$ & $\sim 2000 \mathrm{kbps}$ & $\sim 5000 \mathrm{kbps}$ & $\begin{array}{l}\sim 20 \mathrm{Mbps} \\
(\sim 8 \mathrm{Mbps})\end{array}$ & $\sim 1000 \mathrm{kbps}$ & $\sim 1000 \mathrm{kbps}$ & $\sim 350 \mathrm{kbps}$ & $25 \mathrm{Mbps}$ \\
\hline Audio bitrate & $224 \mathrm{kbps}$ & $\sim 224 \mathrm{kbps}$ & $\sim 448 \mathrm{kbps}$ & $\sim 448 \mathrm{kbps}$ & $\sim 128 \mathrm{kbps}$ & $\sim 128 \mathrm{kbps}$ & $\sim 64 \mathrm{kbps}$ & $\sim 1500 \mathrm{kbps}$ \\
\hline $\begin{array}{l}\text { Size } \\
\text { (MB/min) }\end{array}$ & 10 & $10-20$ & $30-70$ & $\begin{array}{l}\sim 150 \\
(\sim 60)\end{array}$ & $4-10$ & $4-20$ & $2-5$ & 216 \\
\hline CPU Usage & Low & High & Very High & Super high & Very High & High & Low & High \\
\hline Quality & Good & Great* & Excellent* & Superb* & Great* & Great* & Decent* & Excellent \\
\hline
\end{tabular}

kbps = thousand bits per second, Mbps = million bits per second,

approximate bitrate, it can be lower or higher

* the quality of video depends on the video resolution and the bitrate, higher bitrate and higher resolution generally mean better video quality but bigger file size.

\section{Delivery Issues}

\section{a) Content Adaptation}

Generally, content adaptation refers to the reproduction of multimedia content in a different format from a previously produced single version, resulting in the author once present anywhere situation. Nevertheless, according to the findings of the research community, this approach is the most appropriate for multimedia content consumption on mobile devices. This finding can be explained by the nature of most existing multimedia content, which are not designed for handheld mobile devices. Consequently, mobile learners cannot directly utilize most of the learning contents because of incompatibility between the device characteristics they hold and the content utilization requirements. Thus, content adaptation bridges the incompatibility between heterogeneous device characteristics, wireless network dynamics, and diverse multimedia learning content formats. However, creating multiple versions of the same content according to each device and network profile requires tremendous amount of storage; furthermore, such procedure is administratively difficult for the service providers given the different device types and variations in the network characteristics. Figure 4 portrays the entire process pertaining to content adaptation, along with different factors.

\footnotetext{
4 (“Comparison”) ) http://www.videohelp.com/oldguides/comparison
} 


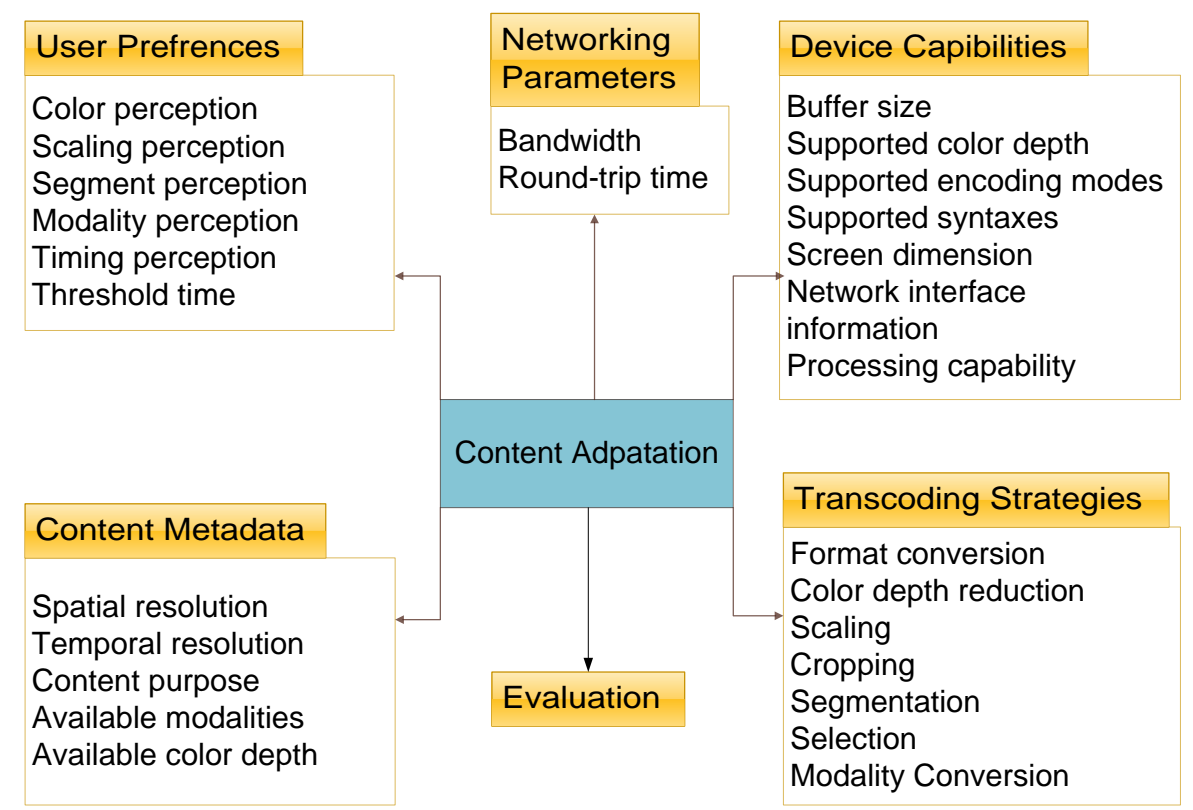

Figure 4 Factors Affecting Content Adaptation Process

\section{i. $\quad$ Device Capabilities}

For an appropriate selection of a suitable content adaptation method, identifying, classifying, and isolating the form factor, computational characteristics, and available software platforms in the learners' mobile device is significantly important. In an HTTP-based m-learning platform, device system identification can be done using the HTTP protocol headers. Otherwise, several other mechanisms can also be utilized based on (1) the W3C "Composite Capability/Preference Profiles (CC/PP)", (2) the "WURFL -Mobile Device Database by ScientiaMobile" XML file and APIs, and (3) the WAP UAPROF (Protocol, 2001).

\section{ii. User Preferences}

Creating a personalized learning content adaptation to satisfy the users' needs require identification and recording. The Dublin Core accessibility and adaptability standard ${ }^{7}$, the profiles of users preferences (Gkatzidou \& Pearson, 2007), and the IMS AccessForAll (Cooper et al., 2005) have attributed the design of a personalized and accessible provisioning of multi-format multimedia contents. With regard to m-learning application, a key accessibility challenge is the variety of user preferences. Furthermore, learning contents must be promptly available to all learners according to their preference. In terms of interaction with or access to the learning contents, disability is redefined as incompatibility between the user preference and the accessible service delivery infrastructure (Cooper et al., 2005).

\section{iii. $\quad$ Content Metadata}

Metadata-driven approaches facilitate the description of the adaptive features of the contents (e.g., what is adapted and what it is adapted to) (Conlan \& Hockemeyer, 2002). The description can be exploited in the client and server sides. For the client side, the best example would be of Synchronized Multimedia Integration Language (SMIL) ${ }^{8}$, a stable and well-known W3C standard client-side adaptation mechanism. The latest specification SMIL defines the semantics and XML syntax for SMIL functionalities. Several straightforward content selection techniques have been defined in SMIL to offer better flexibility and to meet varying contexts of content utilization (including those related to mobile contexts). Thus, the metadata document can provide the mechanisms to perform learning content adaptation,

\footnotetext{
${ }^{5}$ http://www.w3.org/TR/2007/WD-CCPP-struct-vocab2-20070430/

${ }^{6} \mathrm{http}: / /$ wurfl.sourceforge.net/

${ }^{7}$ http://www.imsglobal.org/accessibility/

${ }^{8}$ http://www.w3.org/TR/2008/REC-SMIL3-20081201/
} 
which are supported by the format in modifying the contents to display limitations and bandwidth. In fact, several metadata languages contain an "adaptation" or "alternate content" mechanism (Burnett, Van de Walle, Hill, Bormans, \& Pereira, 2003; Salomoni \& Mirri, 2011).

\section{iv. Network Parameters}

Wireless mobile networks typically suffer from habitual bandwidth fluctuations. Furthermore, network connections are completely unresponsive for several time spans because of the dynamics of the wireless radio network. Given these factors, utilizing several prediction and estimation mechanisms of network parameters is desirable to plan the learning content adaptation process and delivery.

\section{v. $\quad$ Transcoding Strategy}

The transcoding strategy depends on all the four factors explained earlier in this section because they will provide the bases for selecting a transcoding strategy. A key challenge in the transcoding process is the robustness of the transcoding techniques given that multimedia contents are highly real time; the user QoS and QoE are highly dependent on the execution time of the transcoding strategy.

The heterogeneity of the contents and the limitations of the mobile devices and dynamics of the wireless network lead to the adaptation of the contents to facilitate a broad range of users. In fact, the content adaptation process is a computationally expensive task that can either be on the client or server side. For the client side, the mobile device has limited capabilities. Furthermore, downloading a higher amount of data and reducing it afterward are infeasible from the data plan perspective. For server-side transcoding, a scalability limitation exists when the number of users increases. Considering both the cases to maintain an acceptable QoL, exploiting the parallel and abundant computational power of the cloud is necessary using the principles of mobile cloud computing.

\section{vi. Evaluation}

Evaluating the content adaptation process is necessary to ensure that the end mobile learner is receiving the same personalized contents he is looking for (V. Chang, 2006). Furthermore, evaluating and measuring the overall learning process are also necessary to ensure and maintain the QoL. To evaluate the learning content adaptation results, metrics are required to quantify the accuracy of the adaptation process. Precession, recall, and $F$-measure can be utilized as metrics to quantify the accuracy of the content adaptation process given that these metrics are readily applied in information retrieval (Hripcsak \& Rothschild, 2005) and machine learning algorithms (Chau \& Chen, 2008). The definitions of these metrics are as follows:

Precision can be defined as the ratio of appropriately adapted learning contents to the number of all adapted learning contents, expressed as follows:

$$
\text { precision }=\frac{\text { No.of Correctly Adapted Contents }}{\text { No.of Correctly Adapted Contents }}
$$

Recall is the ratio of correctly adapted learning contents to the number of contents delivered, expressed as follows:

$$
\text { recall }=\frac{\text { No.of Correctly Adapted Contents }}{\text { No.of available Contents for adaptation }}
$$

$F$-measure can be presented in terms of precision and recall, as follows:

$$
F-\text { measure }=\frac{2 \times \text { precision } \times \text { recall }}{\text { precision }+ \text { recall }}
$$

In the m-learning session, if all the content adaptation processes in the session can generate a high $F$-measure mean, then their learning session is better in terms of personalized content presentation (Antunes \& Vieira, 2015). If a learning session obtains a precision of 0.8 , then this result indicates that it is $80 \%$ up-to-the expectation of the mobile 
learner. A recall of 0.9 indicates that $90 \%$ of the contents should be adapted in that particular session. The combination of precision, recall, and $F$-measure can be used to determine the QoL in terms of personalized content delivery services.

\section{b) Content Delivery Network}

Content delivery platforms generally fail to have the specific infrastructure, mechanisms, and application data required to generate dynamic learning contents, which multimedia-enabled m-learning applications utilized. Dynamic multimedia contents are provisioned directly from the source of the learning platform and may suffer delays because of WAN latencies. Only a few content delivery services providers, such as Akamai ${ }^{9}$, implement functional and structural components, reducing the delivery and response time of dynamic contents. For the m-learning environment and other multimedia-enabled mobile applications, implementing edge infrastructures over the mobile network operator (MNO) may help mitigate the content delivery issue. The MNO owns all the technical interfaces to enhance the network and speed up content delivery. The lack of interaction and the rational behavior of the actors in distributed mobile application clearly impede a fully operational m-learning ecosystem based on incorporating content delivery functions across the actors and leveraging the monetization of m-learning environments.

\section{User Requirement Issues}

The end user requires the maximum QoE (user perception) and presentation with a minimum monetary cost, apart from the limitations, heterogeneity, and diversity of mobile devices, network, and multimedia contents. Nevertheless, to maximize the end user's QoE, the common solutions suggested by the research community include content adaptation and computational offloading. However, if the techniques are provisioned using a single-server setup, then the application QoS and end user QoE as the number of users and volume of data increase may be degraded. To tackle the situation and allow ubiquitously available cloud computing systems are employed to achieve efficient provisioning. Furthermore, the content delivery clouds are employed to provide scalable access to the contents.

\section{TRENDS IN MULTimedia ENABLED MobiLe LEARNing}

\section{Device Agnostic Multimedia Contents}

With the introduction and widespread acceptance of HTML5, designing interactive/multimedia-enabled learning objects is now possible in nearly all device native formats without utilizing runtimes, such as Adobe Flash Players and Java Applets. With a responsive HTML5 design, modern m-learning applications can automatically adjust to the screen size and resolution of the device. Furthermore, modifying the placement of interacting components in the learning object is possible with such device agnostic design. Device agnostic multimedia contents ensure that every learner subscribed to the m-learning platform receives the same learning experience.

\section{Analytics-Driven Multimedia Learning Contents}

The m-learning systems can generate an enormous amount of data, depending on the scale (geographical and user base) of the learning platform. With the introduction of Big Data analytics, inspecting learner inclinations and improving the m-learning platform are now possible and can help keep track of multimedia contents hotspots, such as identifying which part of a video/audio is mostly viewed/listened and using this information tunes in the learning objects accordingly. Furthermore, analytics can assist in multimedia traffic analysis and automatic grading of students who submit quizzes/exams in the form of audio/video contents.

\section{Augmenting Multimedia Contents through Wearable Technologies}

The advent of wearable technologies, such as Google Glass and Smart Watches and Wear, also paves their way into the realm of m-learning and transforms the way multimedia contents are accessed and acquired. Wearable devices have the ability to turn mockups, models, and simulations into interactive and dynamic learning experiences in $\mathrm{m}$ learning environments. The collaboration of m-learning platform and wearable devices can orchestrate an entire new

\footnotetext{
${ }^{9}$ http://www.akamai.com/cotendo
} 
paradigm of multimedia-enabled m-learning systems. One such orchestration would be to augment the m-learning contents by using an augmented reality device, such as Google Glass.

\section{Internet of Things and Multimedia-Enabled Mobile Learning}

The Internet of Things (IoT) is the next phase of innovation technology and educational applications over the next decade. Despite the increasing capabilities, a number of challenges and limited application framework still exist to utilize these technologies within the m-learning environment. An IoT device can respond to events generated by the m-learning application. In response to such an event, an IoT device can either display/play the learning contents or capture learner responses. The m-learning environments can genuinely become agnostic to time and space if and only if it is integrated with IoT devices.

\section{OPEn ChaLlenges}

\section{Low Complexity Encoding}

The traditional coding techniques are dominated by predictive encoding techniques to achieve high-compression efficiency. These mechanisms are utilized in the ITU-T or the ISO MPEG recommended encoding schemes. The basic idea is to drop the bit rate produced by the source encoding scheme, thereby utilizing source statistics. Therefore, intra-frame compression is employed to minimize repetition in a single frame, while inter-frame compression exploits the correlation among the neighboring frames via motion estimation to minimize the volume of data for storage and transmission. Consequently, the computational complexity required by the motion estimation functionality at the coding side is resource hungry, whereas the decoding units are simple and loaded with low computational overhead.

However, from the information-theoretic bounds for lossy coding (Wyner \& Ziv, 1976) and for lossless coding (Slepian \& Wolf, 1973), an efficient compression mechanism is recognized to be possibly orchestrated by utilizing the information of source statistics at the decoder unit only. Through this methodology, the traditional equilibrium of simple decoder and complex encoder can be retreated (Girod, Aaron, Rane, \& Rebollo-Monedero, 2005). Clearly, such coding mechanisms, where the encoder can be simple and energy efficient, are highly promising for mobile multimedia applications.

\section{Data Dependency}

The parallel computing architecture refers to the simultaneous processing of different independent tasks. This process is generally regarded as an effective and quick method to minimize the processing time and is gradually applied to multimedia processing systems. However, the data dependence multimedia content makes the parallel encoding and decoding complex. As an example, the decoding processing of H.264 has frames of H.264 stream classified into the $\mathrm{I}, \mathrm{B}$, and $\mathrm{P}$ frames. The $\mathrm{P}$ frame constitutes the bitmap data of the I frame, whereas B frame refers to the picture data of the I and P frames. However, such a picture reference decoding will result in data starvation and data collision in the parallel computing architecture.

\section{Measurement and Modeling}

The QoL and QoE associated with mobile multimedia learning applications can be severely affected by the dynamic nature of the wireless network. However, if adaptive mobile multimedia applications are deployed to improve the QoE and QoL, then content adaptation and computational offloading may adversely affect the end user experience because of the intermittent network disruption. Accordingly, developing new quality measuring and modeling techniques is required to ensure proper understanding of the effects of different networks as well as applications, user preferences and mobile device augmentation method and timings.

\section{Security}

Encrypting multimedia contents involves scrambling the multimedia data stream to guarantee secure transmission of the data stream in the client/server transaction. This procedure can be achieved using standard symmetric key cryptographic methods. These methods treat multimedia data stream as a binary stream and cipher the entire stream 
using a traditional cryptographic algorithm, such as AES (Stinson, 2006). In general, non-real-time applications can utilize the traditional encryption algorithms. However, employing standard encryption protocols for the encryption of the complete multimedia data stream is reflected as a naive approach (Agi \& Gong, 1996). A range of limitations exists in the case of multimedia-enabled mobile applications, thereby making such schemes impractical. For example, the available computational resources combined with limited battery life in mobile devices limit the application of AES-like encryptions. Furthermore, encrypting the transmission of multimedia content is beyond the application of the conventional encryption techniques primarily because of the way multimedia is utilized in contrast to textual data. Unlike data encryption, multimedia encryption on mobile device opens various challenging opportunities because the mobile subscribers will not wait for encryption and authentication. Therefore, a secure and real-time streaming of multimedia contents is a serious challenge for mobile multimedia delivery. Judiciously comparing the cost of the protection itself and the cost of the multimedia information requiring protection is necessary to identify an optimal security level for multimedia contents (Pande \& Zambreno, 2011).

From the server side learning environment point of view a recent survey on cloud security states that the security of users' data has the highest priority as well as concern. We believe this can only be able to achieve with an approach that is systematic, adoptable and well-structured such as (Victor Chang, Kuo, \& Ramachandran, 2015; Victor Chang \& Ramachandran, 2015) which has been customized for securing cloud data.

\section{Interoperability}

With the plethora of available mobile device hardware and software architectures, the genuine benefits of the mlearning environment can only be taken when the learning system is designed to handle the heterogeneity of software and hardware platforms. With a heterogeneous learning platform, interoperability in the m-learning systems mainly aims to provide a cohesive and coherent interaction of the subscriber to the learning environment. Standardization, open application programming interfaces, and intermediary layers are few interesting choices of solutions for interoperability (Bernstein, Ludvigson, Sankar, Diamond, \& Morrow, 2009; Kovachev et al., 2011; Salomoni \& Mirri, 2011).

\section{CONCLUSION}

The m-learning platforms revolutionize the life cycle and the way in which the learning process can be augmented with the power of anywhere, anytime computation and communication. The modeling of educational contents (learning objects) for mobile devices and the m-learning platforms is hindered by several pedagogical and technical issues. These issues must be addressed to achieve a seamless learning behavior. As a result, in this study, we presented a taxonomy of technical issues, which can be used as a guideline to design or develop the m-learning environment. Each issue is discussed to provide the reader with brief insights and complexities, thus identifying the causes of these issues. Furthermore, we have identified and briefly discussed several trending areas and how they can improve the multimedia-enabled m-learning paradigm. Finally, we outlined several open research challenges, which must be addressed to improve the overall QoL, user perception, and acceptability of m-learning environments.

\section{ACKNOWLEDGEMENT}

This work is fully funded and partially funded by Bright Spark Program and High Impact Research Grant from the University of Malaya under reference BSP/APP/1635/2013 and UM.C/625/1/HIR/MOE/ FCSIT/03, respectively.

\section{REFERENCES}

AFox, A., Griffith, R., Joseph, A. D., Katz, R., Konwinski, A., Lee, G., ... Zaharia, M. (2010). Above the clouds: A Berkeley view of cloud computing. Communications of the ACM, 53(4), 50-58. Retrieved from http://ftp.cs.duke.edu/courses/cps296.4/compsci590.4/fall13/838-CloudPapers/AboveTheClouds.pdf

Agi, I., \& Gong, L. (1996). An Empirical Study of Secure MPEG Video Transmissions. Network and Distributed System Security, Symposium on, 0, 137. doi:http://doi.ieeecomputersociety.org/10.1109/NDSS.1996.492420 
Ahmad, I., \& Gabbouj, M. (2008). Audio-visual multimedia retrieval on mobile devices. Multimedia Services in Intelligent Environments, 225(2008), 203-225. Retrieved from http://link.springer.com/chapter/10.1007/9783-540-78502-6_8

Alabbadi, M. M. (2011). Cloud Computing for Education and Learning : Education and Learning as a Service ( ELaaS ), (September), 589-594.

Ally, M. (2009). Mobile learning: Transforming the delivery of education and training. Retrieved from http://books.google.com/books?hl=en\&lr=\&id=Itp60WteuJsC\&oi=fnd\&pg=PR1\&dq=Mobile+Learning+Tran sforming+the+Delivery+of+Education+and+Training\&ots=5XKSJ3HRjk\&sig=PK7UEpd4_Leuzx9XrO5wj8pzkI

Ally, M., \& Tsinakos, A. (2014). Increasing Access through Mobile Learning. Perspectives on open and distance learning: increasing access through mobile learning. Retrieved from http://www.col.org/resources/publications/Pages/detail.aspx?PID=466

Antunes, N., \& Vieira, M. (2015). Assessing and Comparing Vulnerability Detection Tools for Web Services: Benchmarking Approach and Examples. IEEE Transactions on Services Computing, 8(2), 269-283. doi:10.1109/TSC.2014.2310221

Bernstein, D., Ludvigson, E., Sankar, K., Diamond, S., \& Morrow, M. (2009). Blueprint for the Intercloud Protocols and Formats for Cloud Computing Interoperability. In 2009 Fourth International Conference on Internet and Web Applications and Services (pp. 328-336). IEEE. doi:10.1109/ICIW.2009.55

Bourguiba, M., Agha, K. Al, \& Haddadou, K. (2012). Improving networking performance in virtual mobile clouds. 2012 Third International Conference on The Network of the Future (NOF), 1-6. doi:10.1109/NOF.2012.6464007

Burnett, I., Van de Walle, R., Hill, K., Bormans, J., \& Pereira, F. (2003). MPEG-21: goals and achievements. IEEE Multimedia, 10(4), 60-70. doi:10.1109/MMUL.2003.1237551

Chang, V. (2006). Web Service Testing and Usability for Mobile Learning. In International Conference on Networking, International Conference on Systems and International Conference on Mobile Communications and Learning Technologies (ICNICONSMCL'06) (pp. 221-221). IEEE. doi:10.1109/ICNICONSMCL.2006.226

Chang, V. (2016). Review and discussion: E-learning for academia and industry. International Journal of Information Management, 36(3), 476-485. doi:10.1016/j.ijinfomgt.2015.12.007

Chang, V., Kuo, Y.-H., \& Ramachandran, M. (2015). Cloud Computing Adoption Framework-a security framework for business clouds. Future Generation Computer Systems, 57, 24-41. doi:10.1016/j.future.2015.09.031

Chang, V., \& Ramachandran, M. (2015). Towards achieving Data Security with the Cloud Computing Adoption Framework. IEEE Transactions on Services Computing, 9(1), 1-1. doi:10.1109/TSC.2015.2491281

Chang, V., Walters, R. J., \& Wills, G. B. (2015). Organisational sustainability modelling-An emerging service and analytics model for evaluating Cloud Computing adoption with two case studies. International Journal of Information Management, 36(1), 167-179. doi:10.1016/j.ijinfomgt.2015.09.001

Chang, V., \& Wills, G. (2013). A University of Greenwich Case Study of Cloud Computing: Education as a Service. (D. Graham, I. Manikas, \& D. K. Folinas, Eds.). IGI Global. doi:10.4018/978-1-4666-3914-0 
Chau, M., \& Chen, H. (2008). A machine learning approach to web page filtering using content and structure analysis. Decision Support Systems. Retrieved from http://www.sciencedirect.com/science/article/pii/S0167923607000875

Chen, X., Liu, J., Han, J., \& Xu, H. (2010). Primary exploration of mobile learning mode under a cloud computing environment. ... , 2010 International Conference on, (095403900), 484-487. Retrieved from http://ieeexplore.ieee.org/xpls/abs_all.jsp?arnumber=5496435

Cloud computing increasingly attractive to universities, says Jisc | Jisc. (n.d.). Retrieved April 26, 2016, from https://www.jisc.ac.uk/news/cloud-computing-increasingly-attractive-to-universities-says-jisc-16-may-2011

Comparison. (n.d.). Retrieved February 10, 2014, from http://www.videohelp.com/oldguides/comparison

Composite Capability/Preference Profiles (CC/PP): Structure and Vocabularies 2.0. (n.d.). Retrieved February 4, 2014, from http://www.w3.org/TR/2007/WD-CCPP-struct-vocab2-20070430/

Conlan, O., \& Hockemeyer, C. (2002). Metadata driven approaches to facilitate adaptivity in personalized eLearning systems. Journal of the Japanese Society for Information and Systems in Education, 38-45. Retrieved from http://css.uni-graz.at/publicdocs/publications/file1083937720.doc

Cooper, M., Hall, W., Keynes, M., Treviranus, J., Library, R., Heath, A., ... Street, P. (2005). Meeting the diversity of needs and preferences - a look at the IMS AccessForAll specifications ' role in meeting the accessibility agenda efficiently, $3-5$.

Deursen, D., Lancker, W., Neve, W., Paridaens, T., Mannens, E., \& Walle, R. (2009). NinSuna: a fully integrated platform for format-independent multimedia content adaptation and delivery using Semantic Web technologies. Multimedia Tools and Applications, 46(2-3), 371-398. doi:10.1007/s11042-009-0354-0

Felemban, M., Basalamah, S., \& Ghafoor, A. (2013). A distributed cloud architecture for mobile multimedia services. IEEE NETWORK, (October), 20-27. Retrieved from http://ieeexplore.ieee.org/xpls/abs_all.jsp?arnumber=6616111

Gao, H., \& Zhai, Y. (2010). System design of cloud computing based on Mobile Learning. ... Acquisition and Modeling (KAM), 2010 3rd ..., 239-242. Retrieved from http://ieeexplore.ieee.org/xpls/abs_all.jsp?arnumber=5646248

Ghazizadeh, A. (2012). Cloud Computing Benefits and Architecture in E-Learning. 2012 IEEE Seventh International Conference on Wireless, Mobile and Ubiquitous Technology in Education, 199-201. doi:10.1109/WMUTE.2012.46

Girod, B., Aaron, A. M., Rane, S., \& Rebollo-Monedero, D. (2005). Distributed video coding. Proceedings of the IEEE, 93(1), 71-83.

Gkatzidou, S., \& Pearson, E. (2007). Vodcasting : A case study in adaptability to meet learners ' needs and preferences, 325-332.

Godwin-jones, R. (2011). EMERGING TECHNOLOGIES MOBILE APPS FOR LANGUAGE LEARNING, 15(2), $2-11$.

He, W., Cernusca, D., \& Abdous, M. (2011). Exploring Cloud Computing for Distance Learning. Online Journal of Distance Learning ..., 1-9. Retrieved from http://www.westga.edu/ distance/ojdla/fall143/he_cernusca_abdous143.html 
Herrington, J., Herrington, A., \& Mantei, J. (2009). Using mobile technologies to develop new ways of teaching and learning. Retrieved from http://researchrepository.murdoch.edu.au/5227/1/Using_Mobile_technologies.pdf

Hripcsak, G., \& Rothschild, A. (2005). Agreement, the f-measure, and reliability in information retrieval. Journal of the American .... Retrieved from https://jamia.oxfordjournals.org/content/12/3/296.full

Juan, S., \& Jun, D. F. (2013). Mobile Learning in the Cloud, 216, 439-446. doi:10.1007/978-1-4471-4856-2

Kitanov, S., \& Davcev, D. (2012). Mobile Learning in Mobile Cloud Computing Environment, 8(December), $27-$ 39. Retrieved from http://www.researchgate.net/publication/248702752_Mobile_Learning_in_Mobile_Cloud_Computing_Enviro nment/file/9c96051e06a16a9b48.pdf

Knoche, H., McCarthy, J., \& Sasse, M. (2005). Can small be beautiful?: assessing image resolution requirements for mobile TV. Proceedings of the 13th Annual .... Retrieved from http://dl.acm.org/citation.cfm?id=1101331

Kovachev, D., Cao, Y., \& Klamma, R. (2011). Mobile Multimedia Cloud Computing and the Web. doi:10.1109/MMWeb.2011.16

Liu, C. (2013). Analysis on the Design Patterns of Cloud Computing in Mobile Learning Systems. Proceedings of the 2nd International Conference on Computer Science and Electronics Engineering (ICCSEE 2013), (Iccsee), 1148-1150. doi:10.2991/iccsee.2013.288

Luo, H., \& Shyu, M.-L. (2011). Quality of service provision in mobile multimedia - a survey. Human-Centric Computing and Information Sciences, 1(1), 5. doi:10.1186/2192-1962-1-5

Masud, M., \& Huang, X. (2012). An e-learning system architecture based on cloud computing. System, 74-78. Retrieved from http://waset.org/journals/waset/v62/v62-15.pdf

Masud, M., \& Huang, X. (2013). A Cloud Based M-learning Architecture for Higher Education. Archives Des Sciences, 66(1), 751-760. Retrieved from http://www.researchgate.net/publication/235758554_A_Cloud_Based_M_learning_Architecture_for_Higher_Education/file/9fcfd5133204440ce0.pdf

Mell, P., \& Grance, T. (2011). The NIST definition of cloud computing (draft). NIST Special Publication, 800(145), 7.

Network Bandwidth Requirements for Media Traffic. (n.d.). Retrieved February 7, 2014, from http://technet.microsoft.com/en-us/library/jj688118.aspx

Pande, A., \& Zambreno, J. (2011). Algorithms for Secure Multimedia Delivery over Mobile Devices and Mobile Agents. ... Multimedia and Mobile Agents: Models .... Retrieved from http://books.google.com/books?hl=en\&lr=\&id=rJuqthLUlNsC\&oi=fnd\&pg=PA232\&dq=Algorithms+for+Sec ure+Multimedia+Delivery+over+Mobile+Devices+and+Mobile+Agents\&ots=6Y3srkL_k_\&sig=r1a_P78enO ZKX-6PxsfqR-w8abw

Protocol, W. A. (2001). WAG UAProf.

Salomoni, P., \& Mirri, S. (2011). Adaptation Technologies in Mobile Learning. Open Source Mobile Learning: Mobile Linux .... Retrieved from http://books.google.com/books?hl=en\&lr=\&id=bTH9WwXCQQIC\&oi=fnd\&pg=PA18\&dq=Adaptation+Tec hnologies+in+Mobile+Learning\&ots=3Qt22lmI-T\&sig=ag0cATwRzz0WLOLbZDp_kTjSbEg 
Sharples, M., \& Roschelle, J. (2010). Guest editorial: Special section on mobile and ubiquitous technologies for learning. ... Transactions on Learning Technologies. Retrieved from http://www.computer.org/csdl/trans/lt/2010/01/tlt2010010004.pdf

Slepian, D., \& Wolf, J. (1973). Noiseless coding of correlated information sources. Information Theory, IEEE Transactions on, 19(4), 471-480.

Stinson, D. R. (2006). Cryptography: theory and practice (Vol. 36). CRC press.

Wheeler, B., \& Waggener, S. (2009). Above-campus services: shaping the promise of cloud computing for higher education. Educause Review. Retrieved from https://www.researchgate.net/profile/Bradley_Wheeler/publication/40832628_Above-

Campus_Services_Shaping_the_Promise_of_Cloud_Computing_for_Higher_Education/links/0fcfd5148736d8 82f8000000.pdf

Wiegand, T., Sullivan, G. J., Bjontegaard, G., \& Luthra, A. (2003). Overview of the H . 264 / AVC Video Coding Standard, 262(July).

WURFL - Mobile Device Database by ScientiaMobile. (n.d.). Retrieved February 4, 2014, from http://wurfl.sourceforge.net/

Wyner, A., \& Ziv, J. (1976). The rate-distortion function for source coding with side information at the decoder. Information Theory, IEEE Transactions on, 22(1), 1-10. 